\title{
Outcome of Intracavitary Electrocardiogram Guidance in PICC Tip Placement in Preterm Infants: A Single-center Experience of 327 Cases
}

\author{
Lilan He, Weiju Chen*, Meng Zhang, Na Lin, Wenyan Yang \\ Neonatal Department, The First Affiliated Hospital of Jinan University, Guangzhou, China
}

Email address:

helilan@yeah.net (Lilan He), chenweiju@126.com (Weiju Chen)

${ }^{*}$ Corresponding author

\section{To cite this article:}

Lilan He, Weiju Chen, Meng Zhang, Na Lin, Wenyan Yang. Outcome of Intracavitary Electrocardiogram Guidance in PICC Tip Placement in Preterm Infants: A Single-center Experience of 327 Cases. American Journal of Nursing Science. Vol. 8, No. 4, 2019, pp. $142-144$. doi: 10.11648/j.ajns.20190804.13

Received: April 5, 2019; Accepted: May 11, 2019; Published: June 4, 2019

\begin{abstract}
A tertiary neonatal intensive care unit (NICU) in China has carried out a study investigating the safety and accuracy of intracavitary electrocardiogram (IC-ECG) guidance in PICC tip placement in preterm infants. From October 2015 to September 2018, IC-ECG-guidance was applied in 327 preterm infants in our NICU. The positioning of the PICC was performed under ECG-guidance and subsequently assessed by chest X-ray. The frequency of correct ECG-guided PICC-placement in one single attempt, premature infants gender, weight, weeks' gestational age at PICC insertion was recorded. 327 preterm infants (207 male and 120 female) were included in the study with a gestation of 31.3 weeks (25.3 to 36.8 ) and birth weight of $1498 \mathrm{~g}$ (600 to 2560). 327 PICCs tip placement was guided with IC-ECG, all concordant with chest X-ray. $313(95.7 \%)$ were correctly positioned, $228(72.3 \%)$ were correctly positioned on the first attempt, 5 were incorrectly identified to have a short line and 9 were malpositioned. The most commonly punctured vein was the basilic vein $(n=228)$, followed by the superficial temporal vein $(n=31)$, the axillary vein $(n=25)$. For 3 years IC-ECG method has proved to be a simple, safe, quick method to assess the correct positioning of the PICC in preterm infants. This is a reliable technique, performing real-time manipulation without any complications.
\end{abstract}

Keywords: Intracavitary ECG, Malposition, PICC, Tip Placement, Premature Infants, Radiography

\section{Introduction}

Peripherally inserted central catheters (PICCs) are being placed routinely to provide nutrition and medications in neonatal intensive care units (NICUs), especially for preterm infants. Insertion of PICCs has become an essential skill in NICU. To date, the main problem inserting a PICC is to determine its position, and the most used method is X-ray, but other radiological and non-radiological techniques are available to both predict the necessary length of insertion and to locate the tip position. In preterm infants, as any possible malposition diagnosed after the procedure would imply a reposition that might be invasive and expensive. For these reasons, the use of the method of intracavitary electrocardiography (IC-ECG) for real-time verification of the tip position appears to be particularly appropriate in preterm infants. Some studys [1, 2, 3] have already demonstrated the safety and feasibility of IC-ECG in the positioning of central venous accesses in adult patients and children $[4,5]$ and have confirmed its accuracy compared with the postoperative chest $\mathrm{X}$-ray. This method has also been used in newborns: Neubauer $[6,7]$ reported the application of this technique in this particular patent population. The author concludes that CVC placement with ECG monitoring is a suitable method to reduce malpositioning; he also reports that there were no side effects specifically related to the ECG method. Although studies describe the use of this method in pediatric patients including infants and neonates. However, few studies regarding PICC placement in preterm infants using IC-ECG technique have been reported. In our study, we assessed the use of IC-ECG for determining PICC tip positions, compared with plain radiographs, in preterm infants in a tertiary NICU. 
In the IC-ECG method for the positioning of the tip, the catheter itself is used as an intracavitary electrode and it can be obtained by two different methods: either the intracavitary electrode is a metallic guide inserted inside the catheter or the intracavitary electrode is a saline solution column contained in the catheter. Within our study, only the saline solution column was considered. As the IC-ECG is based on the variations of the shape of the $\mathrm{P}$ wave, we defined applicability as the percentage of neonates who had a visible $\mathrm{P}$ wave on the surface ECG and thus were eligible for the IC-ECG. The feasibility of the method was defined as the possibility of successfully bringing the procedure described below the "Conclusion" section, which is the proper identification of the "peak" P wave corresponding to the passage between the SVC and RA [8].

\section{Patients and Methods}

Data were prospectively collected from 313 cases of PICC insertions in 327 infants born between October 2015 to September 2018, where was used electrocardiographic guidance to confirm PICC placement. This study was performed in a level III NICU, at a tertiary NICU in Guangzhou, China. The hospital's ethics committee approved the study, and parents or guardians of all enrolled preterm infants provided written informed consents. PICC-NATE $\AA$ $1.9 \mathrm{Fr}$ catheters and Health Line 1.9fr PICC were used. Preterm infants gender, weight at PICC insertion, weeks' postmenstrual age at PICC insertion and day of life at PICC insertion were recorded.

Two neonatal PICC nurses implemented PICC insertion using a 1.9Fr PICC at the bedside. Peripheral veins were selected, and the expected length was estimated on the basis of the surface landmark. Preterm infants received venipuncture and catheterization until the expected length was reached. The electrical transducer is connected both with the catheter and with a cable going to lead II of a standard ECG monitor: the saline column within the catheter acts as an intracavitary electrode. Then, the catheter is filled with saline and connected to the transducer. Then, it is slowly advanced observing the changes of the $\mathrm{P}$ wave till the tip reaches the desired position: as the $\mathrm{P}$ wave gets to its maximal height, the catheter tip is close to the Seno-atrial node, i.e., in the upper part of the right atrium; $1 \mathrm{~cm}$ above this point (when the $\mathrm{P}$ is at one-half of its maximal height), the tip is close to the cavoatrial junction. IC-ECG was induced when the PICC tip reached the point $1 \mathrm{~cm}$ away from the target point based on the expected length. By using a continuous ECG monitor, the insertion depth was regulated until the target $P / R$ wave ratio appeared. In brief, the $\mathrm{P}$ wave became higher when the catheter proceeds into the SVC and reached its peak at the CAJ. Deeper on, a significant reduction in $\mathrm{P}$ wave will appear. Then the catheter was pulled back until the ratio of the amplitudes of $\mathrm{P} / \mathrm{R}$ waves was within the range determined earlier. Finally, the catheter was fastened and a postprocedural chest radiograph was obtained. If the ECG did not appear or its wave shape was disordered during the process, the catheter was pulled back 3 to $4 \mathrm{~cm}$ and reinserted to observe ECG changes again.

\section{Data Collection}

Records of all preterm infants who had a PICC at any time during the study period as identified from our NICU prospectively compiled electronic database were retrieved. Our database has built-in checks with out of range values being flagged immediately. In addition, manual validation occurred annually before the close of the database. Data was collected regarding infant's baseline demographics, details of PICC insertion, type of catheter and details of complications if any.

\section{Results}

From October 2015 to September 2018, IC-ECG-guidance was applied in 327 preterm infants in our NICU. Patient data are summarized in the Table1. 327 preterm infants ( 207 male and 120 female) were included in the study with a gestation of 31.3 weeks ( 25.3 to 36.8 ) and birth weight of $1498 \mathrm{~g}$ (600 to 2560). 327 PICCs tip placement was guided with IC-ECG, all concordant with chest X-ray. $313(95.7 \%)$ were correctly positioned, $228(72.3 \%)$ were correctly positioned on the first attempt, 5 were incorrectly identified to have a short line and 9 were malpositioned. 85 catheters went into an ideal position by withdrawing $0.5-2.5 \mathrm{~cm}$. No side effects or complications associated with IC-ECG occurred. The most commonly punctured vein was the basilic vein $(n=228)$, followed by the superficial temporal vein $(n=31)$, the axillary vein $(n=$ $25)$. The punctured vein is summarized in Table 2.

Table 1. Demographics and results.

\begin{tabular}{ll}
\hline Total & $\mathbf{3 2 7}$ \\
\hline Sex (male/female) & $207 / 120$ \\
Gestational age (wk) & $31.3(25.3$ to 36.8$)$ \\
Weight at insertion $(\mathrm{g})$ & $1498(600$ to 2560$)$ \\
Overall placement success & 313 \\
First attempt success & 228 \\
Malposition & 9 \\
incorrectly positioned & 5 \\
\hline
\end{tabular}

Table 2. Punctured vein.

\begin{tabular}{ll}
\hline basilic vein & $\mathbf{2 2 8}$ \\
\hline axillary vein & 25 \\
superficial temporal vein & 31 \\
others & 29 \\
\hline
\end{tabular}

\section{Discussion}

In the preterm infants, the position of the tip of PICC is most often examined by chest radiography. Confirmation of tip location by post-procedural chest radiography remains acceptable practice and is required in the absence of technology used during the procedure $[9,10]$. Post-procedural radiography is not necessary if alternative tip location 
technology confirms proper tip placement. The IC-ECG method is safe and accurate in pediatric patients as much as in adults. If compared with X-ray, IC-ECG has the obvious advantages of allowing an accurate verification of the tip position, that is, intraprocedural, and without X-ray exposure $[11,12]$.

A few studies describe the use of this method in pediatric patients including infants and neonates while supporting data in preterm infants are still lacking. In the study by Zhou and colleagues, [8-9] they refer to neonates of higher gestational age and weight (mean gestational age and mean birth weight of 35.4 weeks and $2629 \mathrm{~g}$ vs 29.4 weeks and $1213 \mathrm{~g}$, respectively). In fact, even in the work by Capasso $A$ and colleagues [13], they refer to gestational age 29.41 \pm 3.04 (range: $25-40$ ) weeks. Furthermore, until now, this is the first study of IC-ECG on the catheter's tip position on preterm infants. Our study demonstrated the outcome of intracavitary electrocardiogram guidance in PICC tip placement in preterm infants. The accuracy of the method is $95.7 \%$. No side effects were registered during procedures for tip location.

The most commonly punctured veins in our study were basilic veins, which is consistent with NANN's recommendation. Our hospital is a tertiary hospital, and most of the hospitalized patients in our NICU is preterm infants. Therefore, the recommended veins for PICC, such as basilica veins and superficial temporal veins, were not acupunctured and damaged usually. Superficial temporal veins (mainly right) were secondly used for cannulation. These veins are visualized, fixed, and observed easily after catheterization. But the risk of resistance to threading can occur when the catheter traverses the area in front of the ear and when it enters the subclavian vein, thus decreasing the success rate of PICC placement. The third punctured veins were axillary veins, because of the large size and short distance to the SVC, so these veins annulate easier.

In our study, all of the 327 preterm infants whose PICCs had been successfully placed and correct tip location had been verified by chest radiography acquired stable and legible ECG and $\mathrm{P}$ wave on IC-ECG. Identification of the peak of the $\mathrm{P}$ wave during the maneuver; the final position of the tip (about half to one-third of the maximum amplitude; this will correspond to the lower third of the SVC). We applied IC-ECG guided PICC catheterization with the target point in 313 preterm infants. The results showed that $95.7 \%$ of IC-ECG guided PICC catheterization in preterm infants lead to correct tip location in the first attempt, similar to other reports $[14,15]$.

\section{Conclusion}

In conclusion, for 3 years IC-ECG method has proved to be a simple, safe, quick method to assess the correct positioning of the PICC in preterm infants. This is a reliable technique, performing real-time manipulation without any complications.

\section{References}

[1] Pittiruti M, Bertollo D, Briglia E, et al. The intracavitary ECG method for positioning the tip of central venous catheters: results of an Italian multicenter study [J]. Journal of Vascular Access, 2012, 13 (3): 357-365.

[2] Oliver G, Jones M. ECG-based PICC tip verification system: an evaluation 5 years on. [J]. British Journal of Nursing, 2016, 25 (19): S4-S10.

[3] Walker G, Chan R J, Alexandrou E, et al. Effectiveness of electrocardiographic guidance in CVAD tip placement [J]. British Journal of Nursing, 2015, 24 (14): S4, S6, S8.

[4] Perin G, Scarpa M G. Defining central venous line position in children: tips for the tip [J]. Journal of Vascular Access, 2016, $16(2): 0-0$.

[5] Rossetti F, Pittiruti M, Lamperti M, et al. The intracavitary ECG method for positioning the tip of central venous access devices in pediatric patients: results of an Italian multicenter study. [J]. Journal of Vascular Access, 2014, 13 (3): 137-143.

[6] Neubauer A P. Central venous placement of silastic catheters by a recording of an intravascular ECG--a prospective study in 50 premature infants weighing less than $1000 \mathrm{~g}$. [J]. Klinische Padiatrie, 1991, 203 (3): 146-148.

[7] Neubauer A P. Percutaneous central i.v. access in the neonate: experience with 535 silastic catheters [J]. Acta Paediatrica, 2010, 84 (7): 756-760.

[8] Weber F, Buitenhuis M, Lequin M H. Determination of the optimal length of insertion of central venous catheters in pediatric patients by endovascular ECG [J]. Minerva Anestesiologica, 2013, 79 (4): 379-84.

[9] Perin G. PICC placement in the neonate [J]. New England Journal of Medicine, 2014, 370 (22): 2153.

[10] Sharpe E, Kuhn L, Ratz D, et al. Neonatal Peripherally Inserted Central Catheter Practices and Providers: Results From the Neonatal PICC1 Survey [J]. Advances in Neonatal Care, 2016, 17: 1 .

[11] Gemma O, Matt J. ECG or X-ray as the 'gold standard' for establishing PICC-tip location? [J]. British Journal of Nursing, 2014, 19: 10-6.

[12] Baldinelli F, Capozzoli G, Pedrazzoli R, Marzano N. Evaluation of the correct position of peripherally inserted central catheters: anatomical landmark vs. electrocardiographic technique [J]. Journal of Vascular Access, 2015; 16 (5): 394-398.

[13] Capasso A, Mastroianni R, Passariello A, et al. The intracavitary electrocardiography method for positioning the tip of epicutaneous cava catheter in neonates: Pilot study [J]. The Journal of Vascular Access, 2018:112972981876129.

[14] Zhou L, Xu H, Liang J, et al. Effectiveness of Intracavitary Electrocardiogram Guidance in Peripherally Inserted Central Catheter Tip Placement in Neonates [J]. Journal of Perinatal \& Neonatal Nursing, 2017, 31 (4).

[15] Zhou L, Xu H, Xu M, et al. An accuracy study of the Intracavitary Electrocardiogram (IC-ECG) guided peripherally inserted central catheter tip placement among neonates [J]. Open Med, 2017, 12 (1): 125-130. 